\title{
Chemotherapy Plus Bevacizumab as Neoadjuvant or Conversion Treatment in Patients with Colorectal Liver Metastases
}

\author{
PILAR GARCÍA ALFONSO ${ }^{1}$, MERCEDES CAVANAGH PODESTA ${ }^{1}$, ANDRÉS MUÑOZ MARTÍN ${ }^{1}$, \\ MONTSERRAT BLANCO CODEISIDO ${ }^{1}$, AITANA CALVO ${ }^{1}$, ISABEL PELIGROS ${ }^{2}$, \\ ANA CORCUERA ${ }^{1}$, ANA BELÉN RÚPEREZ BLANCO ${ }^{1}$, SARA CUSTODIO-CABELLO ${ }^{1}$, \\ DANIEL LÓPEZ TRABADA ${ }^{1}$, MIGUEL MARTÍN ${ }^{1}$ and ENRIQUE DE RAMÓN ${ }^{3}$ \\ Departments of ${ }^{1}$ Medical Oncology, ${ }^{2}$ Anatomical Pathology, and ${ }^{3}$ Radiology, \\ Gregorio Marañon University General Hospital, Madrid, Spain
}

\begin{abstract}
Aim: To evaluate the efficacy of chemotherapy plus bevacizumab as neoadjuvant or conversion treatment for colorectal liver metastases (CLM). Patients and Methods: A retrospective chart review was carried out of 74 patients with CLM treated with neoadjuvant or conversion chemotherapy plus bevacizumab. Results: The overall response rate was $63.4 \%$. An optimal morphological response by computed tomography was reported in 35\% of patients. The rate of complete resection was $71.6 \%$. Complete or major pathological response $(p R)$ was attained in $58.2 \%$. The median overall survival (OS) was not reached. Median progression-free (PFS) and relapse-free (RFS) survival were 14.6 and 8.7 months. Among patients reaching an optimal $p R$, median $O S$ was not reached ( $p=0.08)$, and a trend towards longer RFS and PFS was seen. Conclusion: Neoadjuvant or conversion chemotherapy with bevacizumab is an active and tolerable option for CLM with minimal postsurgery complications. Optimal $p R$ is associated with a longer $O S$ and a trend for prolonged PFS and RFS.
\end{abstract}

Colorectal cancer (CRC) is a major cause of death due to cancer worldwide and the second leading cause of cancer mortality in Europe (1). The liver is the most common metastatic site for CRC, with approximately $25 \%$ of patients presenting with liver metastases at diagnosis and up to $50 \%$ over the course of their disease. Surgical resection is

Correspondence to: Dr. Pilar García Alfonso, Servicio de Oncología Médica, Hospital General Universitario Gregorio Marañón, Calle del Dr. Esquerdo, 46, 28007 Madrid, Spain. Tel: +34 915868115, e-mail: pgarcaalfonso@gmail.com

Key Words: Bevacizumab, chemotherapy, colorectal cancer, liver metastases, pathological response. considered the only potentially curative treatment for patients with resectable colorectal liver metastases (CLM) and provides significant improvement of overall survival (OS) $(2,3)$. However, only a small proportion of patients have resectable disease at presentation (4).

Preoperative chemotherapy combined with the biological targeted agent bevacizumab allows tumor downsizing, thereby increasing the proportion of patients with unresectable CLM who are subsequently able to undergo liver resection $(3,5,6)$. Adding bevacizumab to standard chemotherapy has been demonstrated to improve OS (7) and progression-free survival (PFS) $(7,8)$ compared with chemotherapy alone in patients with metastatic CRC. Moreover, several retrospective studies have demonstrated higher optimal pathological response $(\mathrm{pR})$ rates with preoperative bevacizumab plus chemotherapy than with chemotherapy alone after surgical resection (9-12). However, these studies used heterogeneous methods and criteria to assess $\mathrm{pR}$. In addition, there are currently limited prospective data on $\mathrm{pR}$ after CLM resection following preoperative bevacizumab plus chemotherapy treatment (13).

Despite the clear survival advantage of hepatic resection of CLM, relapse is common (14). Clinical factors are insufficient to stratify prognosis after CLM resection. Therefore, in the era of tailored therapy, identification of markers with prognostic value is an urgent clinical need in order to optimize the clinical management of patients eligible for bevacizumab-based preoperative treatment. Pathologic response is considered a relevant prognostic factor of survival after CLM resection following preoperative chemotherapy $(9,15-17)$. There is now reasonable evidence to suggest that $\mathrm{pR}$ may be an appropriate surrogate marker of long-term efficacy for targeted agents although limited data are available on its correlation with survival (11). 
This chart review study assessed the efficacy of bevacizumab plus chemotherapy as neoadjuvant or conversion treatment in terms of Response Evaluation Criteria In Solid Tumors (RECIST) (18), morphological response by computed tomography (CT), pR and long-term outcome in patients with CLM under clinical practice conditions. The association of $\mathrm{pR}$ following preoperative bevacizumab treatment with survival was also evaluated.

\section{Patients and Methods}

Study design and patient population. This was a retrospective chart review conducted at a Spanish University Hospital. The study was carried out in accordance with the Declaration of Helsinki, all its amendments and national regulations. The project was approved by the Ethics Committee of the University General Hospital Gregorio Marañón (Madrid) (approval number 392/13), which waived the requirement for informed consent for retrospective collection of data from patients' medical charts.

Records for consecutive patients aged $\geq 18$ years with CLM who had received first- or second-line neoadjuvant or conversion chemotherapy plus bevacizumab before undergoing hepatic resection between 2005 and 2014 were retrospectively reviewed. Data collection from patients' hospital records included demographic and clinical data, surgery-related data and clinical management, and outcome.

The primary study endpoint was effectiveness of bevacizumab plus chemotherapy in terms of response based on morphological changes observed on computed tomography, RECIST-based response and $\mathrm{pR}$ after CLM resection and also based on OS, PFS and relapse-free survival (RFS). In addition, we evaluated the PFS, RFS and OS stratified by $\mathrm{pR}$. The relationship between morphologic or RECIST response and $\mathrm{pR}$ was also assessed. Clinical response was assessed based on RECIST criteria (18). The overall response rate (ORR) was calculated as the sum of patients reaching a complete (CR) or partial (PR) response as the best response achieved during neoadjuvant or conversion treatment. Patients with $\mathrm{CR}$ and PR were classified as responders and patients with stable (SD) or progressive (PD) disease were defined as non-responders.

Pathological response was evaluated using previously determined cutoff values for the percentage of residual tumor cells (9), and categorized as complete (no tumor cells), major $(<50 \%$ residual tumor cells), or minor ( $\geq 50 \%$ residual tumor cells). Optimal response was defined as complete or major $\mathrm{pR}$ and suboptimal $\mathrm{pR}$ corresponded to minor response.

Morphological response was assessed using new morphological criteria (19) and defined as optimal when metastases changed from group 3 (heterogeneous attenuation with a thick, poorly defined tumor-liver interface) or 2 (intermediate morphology that cannot be rated as group 1 or 3 ) to 1 (homogeneous low attenuation with a thin, sharply defined tumor-liver interface) or suboptimal (incomplete, if the group changed from 3 to 2, and none if the group number had not changed or increased). CT scans were performed with 16 slice multidetector-row CT (Brilliance; Philips Medical Systems, Eindhoven, the Netherland). Images were obtained at the portal venous phase of enhancement after injection of $125 \mathrm{ml}$ of nonionic contrast medium (Iopamiro 300; Bracco, Milan, Italy) with a power injector at a rate of $3 \mathrm{ml} / \mathrm{s}$. Imaging commenced $70 \mathrm{~s}$ after the start of the injection. Response evaluation was reviewed by a radiologist who was blinded to pathological findings and patient treatment.

In the absence of a standardized definition for resectability, it was assessed by a multidisciplinary team including a liver surgeon, a radiologist and a medical oncologist. Unresectable metastases were those fulfilling at least one of the following criteria: no possibility of upfront R0 resection of all hepatic lesions; $<30 \%$ estimated residual liver after resection; metastases in contact with major vessels of the remnant liver. Patients with CLM and concomitant extra-hepatic disease (EHD) were selected for surgery if they had limited disease (lung or lymph node metastases, and up to five lesions) and potentially resectable lesions.

Statistical analysis. A descriptive statistical analysis was performed on study variables. Continuous variables were compared using the Mann-Whitney $U$-test, and categorical variables were compared using the chi-squared or Fisher's exact test.

OS was measured from the date of hepatic resection to the date of death. RFS was calculated as the time from hepatic resection until documented recurrence or death from any cause. PFS was calculated as the time from neoadjuvant or conversion treatment initiation until documented recurrence or death from any cause. The probability of PFS, RFS and OS was estimated using the Kaplan-Meier method. Log-rank tests were used to compare survival curves of different subgroups. All patients who had received at least one dose of study treatment were included in the safety analysis. The statistical analysis was performed using the Statistical Package for the Social Sciences (SPSS) version 18.0 (SPSS Inc, Chicago, IL, USA).

\section{Results}

Patient characteristics. Of 86 eligible patients, 12 were excluded from the analysis due to non-fulfillment of eligibility criteria: 11 did not receive bevacizumab plus chemotherapy immediately before surgery and one did not undergo hepatic resection and received radiotherapy instead. The demographic and clinical characteristics of the 74 patients finally analyzed are shown in Table I.

The median time from diagnosis of metastatic disease to surgery was 7.9 (range=6.0-11.6) months. Of patients with synchronous primary tumor $(n=53), 92.2 \%$ underwent surgery, which was performed before resection of CLM in $71.7 \%$ of these patients. All patients underwent CLM resection, with a median number of 6 (range $=5-8$ ) metastases resected. Onestage liver surgery was performed in most patients $(83.8 \%)$, while nine $(12.2 \%)$ patients underwent two-stage hepatectomy. Portal vein embolization was performed in five $(6.8 \%)$ patients. A total of $21(29.2 \%)$ patients underwent radiofrequency ablation for CLM. The median number of lesions treated by radiofrequency was 2 (range $=1-3$ ).

Surgical resection of CLM was performed after a median of 6 (range $=5-8$ ) weeks from the last dose of bevacizumab and 5 (range=4-7) weeks after the last dose of any drug administered. Sixty-three $(85.1 \%)$ and 10 patients $(13.5 \%)$ received a regimen containing bevacizumab as first- or second-line treatment prior to hepatic resection, respectively. The most frequent regimen used in combination with 
Table I. Patient demographic and clinical characteristics $(n=74)$.

\begin{tabular}{lc}
\hline Variable & Value \\
\hline Age at surgery (range), years & \\
$\quad$ Median (range) & $62(53-70)$ \\
Gender, $\mathrm{n}(\%)$ & \\
Male & $41(55.4)$ \\
Female & $32(43.2)$ \\
Missing & $1(1.4)$ \\
ECOG PS at diagnosis, $\mathrm{n}(\%)^{\mathrm{a}}$ & \\
0 & $23(31.1)$ \\
1 & $51(68.9)$ \\
Primary tumor, $\mathrm{n}(\%)$ & \\
Colon & $38(51.4)$ \\
Rectum & $18(24.3)$ \\
Colon and rectum & $9(12.2)$ \\
Missing data & $9(12.2)$ \\
Tumor disease presentation, $\mathrm{n}(\%)$ & \\
Synchronous & $53(71.6)$ \\
Metachronous & $18(24.3)$ \\
Missing data & $3(4.1)$ \\
Location of metastases, $\mathrm{n}(\%)^{\mathrm{b}}$ & \\
Liver-only & $46(62.2)$ \\
Extra-hepatic & \\
Local/regional & $28(37.2)$ \\
Lung & $13(17.6)$ \\
Other & $15(20.3)$ \\
Multiple sites & $11(14.9)$ \\
Resectability of metastases $\mathrm{n}(\%)$ & $4(5.4)$ \\
Initially resectable & \\
Potentially resectable & $8(10.8)$ \\
Unresectable & $56(75.7)$ \\
Missing & $8(10.8)$ \\
KRAS mutation status, $\mathrm{n}(\%)$ & $2(2.7)$ \\
Wild-type & \\
Mutant & $37(56.9)$ \\
\hline
\end{tabular}

ECOG PS: Eastern Cooperative Oncology Group Performance Status; 5-FU/LV: 5-fluorouracil/leucovorin. Percentages may not add up to $100 \%$ due to rounding. aAt diagnosis of metastatic disease. bSome patients had more than one metastatic site. ${ }^{\mathrm{c}} \mathrm{At}$ least one metastatic site other than liver; only one patient had three sites.

bevacizumab was fluorouracil-based chemotherapy doublets including oxaliplatin $(56.8 \%)$ and irinotecan $(23.1 \%)$ (Table II). Patients received a median of 7 (range $=5-10)$ chemotherapy cycles. The median time on first-line neoadjuvant or conversion treatment was 3.7 (range=2.3-5.6) months. About three-quarters of all patients received adjuvant chemotherapy after CLM resection. Among these, nearly $80 \%$ of patients received bevacizumab-based adjuvant therapy (Table II). Patients received a median of 6.0 (range=4.0-8.0) cycles during a median of 3 (range=2.5-4.0) months.

Clinical outcome. The summary of efficacy data is shown in Table III. The ORR to preoperative treatment with
Table II. Neoadjuvant or conversion first- and second-line treatment with chemotherapy plus bevacizumab and adjuvant treatment after resection of colorectal liver metastases.

\begin{tabular}{lc}
\hline Regimen & $\mathrm{N}(\%)$ \\
\hline Neoadjuvant or conversion treatment & \\
5-FU/LV + oxaliplatin & $35(47.3)$ \\
5-FU/LV + irinotecan & $12(16.3)$ \\
Capecitabine + oxaliplatin & $7(9.5)$ \\
5-FU/LV + oxaliplatin + irinotecan & $6(8.1)$ \\
Capecitabine + irinotecan & $5(6.8)$ \\
5-FU/LV & $2(2.7)$ \\
5-FU/LV + oxaliplatin + cetuximab & $1(1.4)$ \\
Second-line treatment (n=10) & \\
5-FU/LV + oxaliplatin + cetuximab & $3(4.1)$ \\
5-FU/LV + irinotecan + irinotecan & $1(1.4)$ \\
Capecitabine + cetuximab & $1(1.4)$ \\
5-FU/LV + oxaliplatin & $1(1.4)$ \\
Adjuvant treatment after liver resection & \\
Total & $56(75.7)$ \\
Bevacizumab plus chemotherapy & $44(78.6)$ \\
Bevacizumab + 5-FU/LV + oxaliplatin & $18(32.1)$ \\
Bevacizumab + 5-FU/LV + irinotecan & $9(16.1)$ \\
Bevacizumab + capecitabine + oxaliplatin & $5(8.9)$ \\
Bevacizumab + 5-FU/LV & $4(7.1)$ \\
Bevacizumab + capecitabine & $3(5.4)$ \\
Othera & $5(9.0)$ \\
\hline
\end{tabular}

5-FU/LV: 5-Fluorouracil/leucovorin aBevacizumab-based adjuvant treatment administered to $<5 \%$ of patients (therapies received by one or two patients each).

bevacizumab was $63.4 \%$. None of the patients achieved CR by RECIST. Out of 74 evaluable patients, 34 (46\%) were unsuitable for radiological evaluation by CT because of lowquality images. Therefore, CT-based response was assessed in $40(54 \%)$ patients. Of these, $15(37.5 \%)$ attained an optimal morphological response.

R0 resection was achieved in $71.6 \%$ of patients. The eight patients with initially unresectable metastases underwent complete resection of CLM. A complete or major $\mathrm{pR}$ was achieved by nearly $60 \%$ of patients.

EHD was resected in all patients after surgical resection of CLM $(n=28)$. Eight $(53.3 \%)$ patients underwent surgical resection of lung metastases, and all local/regional metastases $(n=13)$ and other extrahepatic metastases $(n=11)$ were resected. Resection of EHD was complete (R0) in all patients.

With a median duration of follow-up of 35.7 (range=20.053.2) months, median OS was not achieved. The 5-year OS was nearly $60 \%$, and $66.2 \%(n=49)$ of patients were alive at the time of analysis. Sixty-one (82.4\%) patients had experienced relapse. The median RFS was 8.7 (95\% confidence interval=5.7-11.6) months. Median PFS was 14.6 (95\% confidence interval=10.8-18.5) months (Figure 1). 
There were no significant differences in the clinical outcome between patients with liver-only metastases and those with EHD, although a trend for a longer RFS (9.4 vs. 7.4 months; $p=0.089$ ), DFS (15.4 vs. 12.7 months; $p=0.078$ ) and OS (not reached $v s .40 .3 ; p=0.158$ ) was seen in patients with liver-only disease.

A significantly longer RFS and a trend for a prolonged PFS was achieved in patients who received adjuvant treatment after hepatic resection compared with those who did not (RFS: 9.5 vs. 3.5 months; $p=0.001$; PFS: 16.9 vs. 9.1 months; $p=0.079)$. The median OS was not achieved irrespective of the administration of adjuvant therapy $(p=0.480)$.

Association of pathological response with PFS and OS. While the median OS in patients with a minor response was 37.4 months (5-year OS $=45.8 \%$ ), it was not reached for patients reaching an optimal pR (5-year OS=67.9\%) $(p=0.08)$. Similarly, a trend to a longer PFS and RFS was seen in patients who achieved an optimal pR (PFS: $16.8 v s$. 12.7 months, $p=0.201$; RFS: $9.4 v s .6 .2$ months, $p=0.107$ ) (Figure 2).

Association between morphological, RECIST and pathological response. Patients achieving a suboptimal morphological response were more likely to attain a minor pR compared with RECIST non-responders [12/13 (92.3\%) vs. 4/13 (30.8\%); $p=0.019]$. However, the proportion of patients who achieved a complete or major $\mathrm{pR}$ was similar between morphologic and RECIST responders [14/26 (53.8\%) vs. 17/26 (65.4\%); $p=0.415]$.

Clinical outcome according to KRAS mutation status. The ORR was $68.4 \%$ and $50.0 \%$ in patients with wild-type $K R A S$ and in those carrying KRAS mutations, respectively $(p=0.130)$. The median OS for patients with KRAS-mutated tumors was 37.4 months and was not reached in those with wild-type $K R A S(p=0.496)$. Similarly, patients with wild-type $K R A S$ tumors attained a longer, albeit nonsignificant, median PFS and RFS compared with those carrying KRAS mutations (15.4 vs. 11.4, $p=0.326 ; 10.5$ vs. 6.2 months; $p=0.311$ ).

Safety. All patients included in the study were considered evaluable for safety analyses $(n=74)$. Thirteen patients experienced at least one adverse event. A total of $61(82 \%)$ did not suffer any grade $3 / 4$ adverse event before surgery. The most frequent grade $3 / 4$ adverse events were neutropenia and diarrhea in four patients each.

Postoperative complications. The median hospitalization was 9 (range=6-16) days. Thirty patients (40.5\%) suffered from at least one postoperative complication. Intra-abdominal infection $(\mathrm{n}=6,8.1 \%)$, pneumonia $(\mathrm{n}=6,8.1 \%)$ and wound
Table III. Summary of efficacy outcomes for neoadjuvant or conversion chemotherapy plus bevacizumab.

\begin{tabular}{lc}
\hline Variable & Value \\
\hline RECIST response, $\mathrm{n}(\%)$ & \\
Complete & $0(0.0)$ \\
Partial & $45(60.8)$ \\
Stable disease & $24(32.4)$ \\
Disease progression & $2(2.7)$ \\
Unknown & $3(4.1)$ \\
Overall response rate & $45(63.4)$ \\
95\% CI & $51.1-74.3$ \\
Morphological response, $\mathrm{n}(\%)$ & \\
Optimal & $14(18.9)$ \\
Incomplete & $11(14.9)$ \\
None & $15(20.3)$ \\
Not available & $34(45.9)$ \\
Resection, $\mathrm{n}(\%)$ & $53(71.6)$ \\
Complete (R0) & $19(25.7)$ \\
Incomplete (R1) & $2(2.7)$ \\
Unknown & \\
Pathological response, $\mathrm{n}(\%)$ & $5(6.8)$ \\
Complete & $38(51.4)$ \\
Major & $26(35.1)$ \\
Minor & $5(6.8)$ \\
Unknown &
\end{tabular}

CI: Confidence interval; RECIST: Response Evaluation Criteria in Solid Tumors (18).

infection $(\mathrm{n}=5,6.8 \%)$ were the most frequent complications followed by anastomotic leak $(\mathrm{n}=2,2.7 \%)$, hepatic failure $(\mathrm{n}=2,2.7 \%)$, reintervention $(\mathrm{n}=2,2,7 \%)$, bile leak $(\mathrm{n}=2$, $2.7 \%)$, and paralytic ileus $(\mathrm{n}=2,2.7 \%)$. There were no deaths due to complications of surgery.

Hepatotoxicity. Thirty $(40.5 \%)$ patients experienced postsurgery liver toxicity at least once. The most frequently reported hepatic toxicities were steatohepatitis (20.3\%) and sinusoidal dilatation (14.9\%). Among 11 patients with sinusoidal dilatation, nine (82\%) patients had received firstor second-line oxaliplatin-based chemotherapy. Of 15 patients experiencing steatohepatitis, six $(40 \%)$ had been treated with irinotecan.

\section{Discussion}

This retrospective chart review provides additional evidence supporting the benefit of neoadjuvant or conversion bevacizumab with chemotherapy in terms of tumor response, $\mathrm{pR}$ and long-term outcome after CLM resection in clinical practice. Additionally, this strategy was demonstrated to be tolerable, with minimal post-surgery complications. Of note, our findings confirm the association between $\mathrm{pR}$ and $\mathrm{OS}$ after CLM resection. 

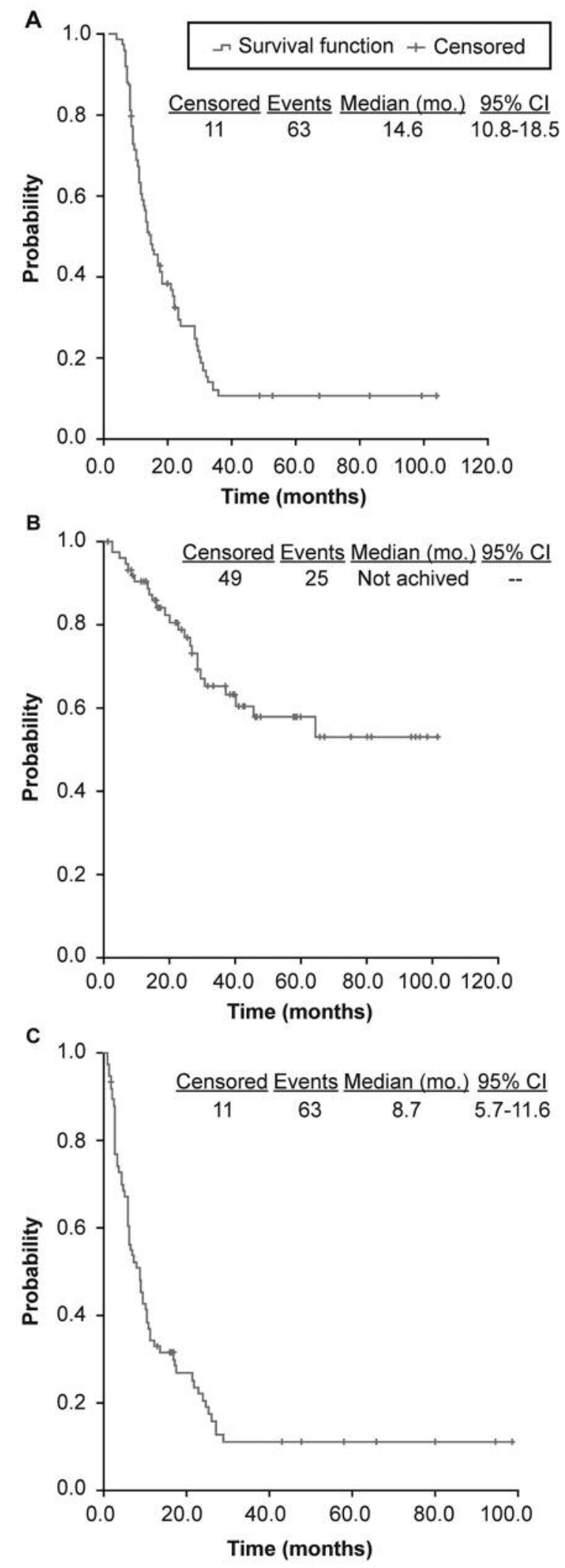

Figure 1. Kaplan-Meier curves for progression-free (A), overall $(B)$ and relapse-free (C) survival. $95 \%$ CI: 95\% Confidence interval; mo.: months.
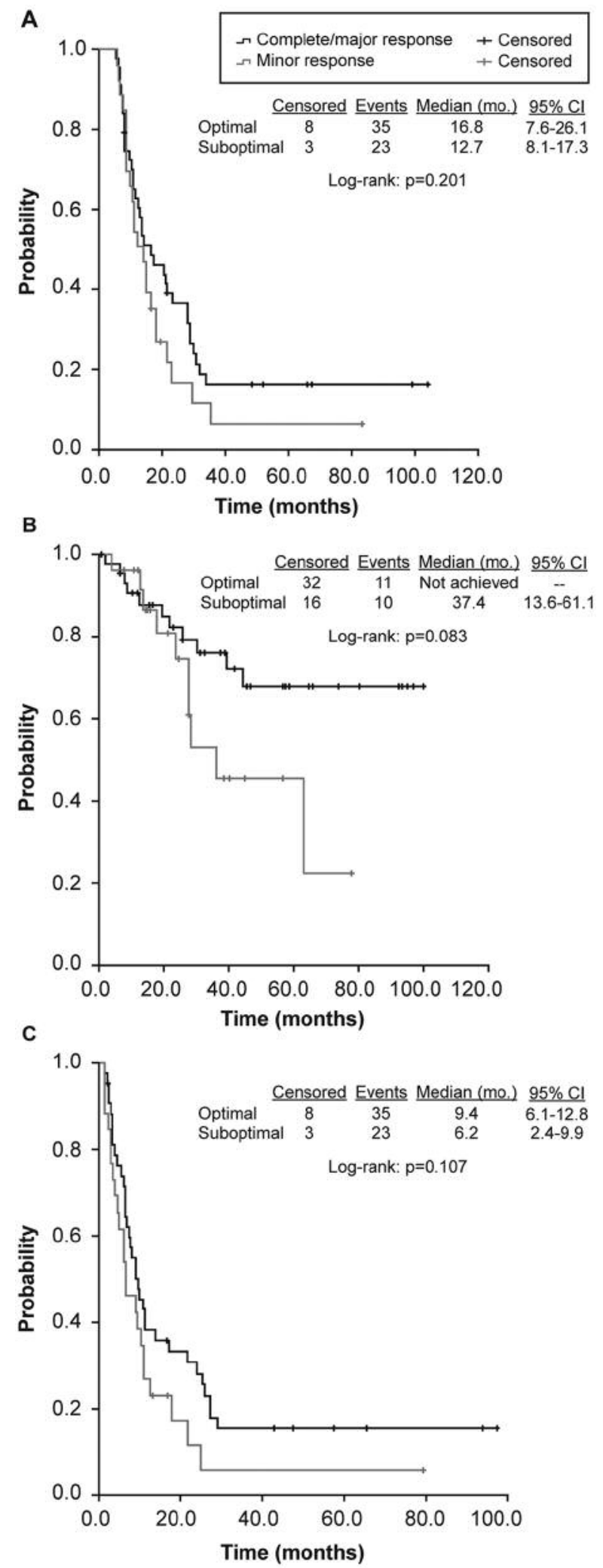

Figure 2. Kaplan-Meier curves for progression-free $(A)$, overall $(B)$ and relapse-free $(C)$ survival according to pathological response (optimal vs. suboptimal response). 95\% CI: 95\% Confidence interval; mo.: months. 
The RECIST ORR achieved after neoadjuvant or conversion bevacizumab treatment $(63 \%)$ was similar to previous retrospective and prospective experience on the efficacy of preoperative bevacizumab in patients with CLM $(13,20,21)$. It is worth noting, as a descriptive comparison only, that the ORR was comparable to that reported in the phase II OLIVIA study in selected patients with liver-limited disease, in whom bevacizumab plus chemotherapy seems to be more effective (13).

The median PFS noted in this analysis lies within the range of that reported in previous phase II studies with preoperative bevacizumab plus chemotherapy regimens in patients with unresectable liver-only metastases $(6,13,22)$. In addition, the PFS benefit is also comparable to that obtained with cetuximab plus chemotherapy (23) and with panitumumab (24) in patients with unresectable liver-limited disease. Median time to OS after hepatic resection was not reached in our series. This favorable impact on survival is in line with that reported in the FOLFOX arm of the phase II OLIVIA study (13). Notably, our results also confirm the RFS attained in the bevacizumab plus FOLFOX arm in the aforementioned study (13).

It is noteworthy that the clinical outcome data shown in this study, with approximately $40 \%$ of patients with EHD, are similar to those reported in previous studies wherein only patients with liver-limited disease were included $(6,13,22)$. Although the presence of EHD has traditionally been considered a contraindication to liver resection (25), evidence has emerged regarding the improvement of prognosis when hepatic resection is performed in patients with CLM and limited EHD (26-29). Resection of CLM when EHD is present can result in a similar survival rate to that obtained in patients with liver-only metastases (30). In our study, it must be taken into account that most patients with EHD had only one site of metastasis and lung metastases, which has been associated with a similar survival to liver-only metastases (31).

The clinical benefit of bevacizumab in metastatic $\mathrm{CRC}$ has been shown to be independent of KRAS mutation status (8, 32). However, other studies suggest a correlation of KRAS mutation with a higher risk of recurrence and poorer survival in patients undergoing CLM resection (33). Accordingly, we found a trend for a superior, although not significant benefit in terms of ORR, OS and RFS, in those patients carrying $K R A S$ wild-type tumors.

The major and complete $\mathrm{pR}$ rates achieved in our series were comparable to those reported in the bevacizumab plus oxaliplatin-based arm of previous retrospective studies where the same criteria for $\mathrm{pR}$ assessment were used $(9,10)$. The optimal pR benefit was also consistent with the only prospective study evaluating histopathological response after CLM resection following preoperative bevacizumab treatment (57\%) (13). However, comparison with previous studies (9-12) is limited by the heterogeneity in the methods and criteria used to assess $\mathrm{pR}$.

Pathological response to preoperative chemotherapy has been demonstrated to correlate with improved survival (9). However, there are limited data regarding the potential correlation between $\mathrm{pR}$ and survival when preoperative bevacizumab is added to chemotherapy (11). Our findings showed that optimal $\mathrm{pR}$ is associated with a longer OS compared with those with minor or no response (median OS not achieved vs. 37.4 months). Although this was not statistically significant according to the conventional threshold of significance $(p<0.05)$, a clear long-term benefit was obtained in patients achieving optimal $\mathrm{pR}$, therefore confirming the limited experience in this regard (11). More recently, poorer PFS and OS have been associated with low grade of $\mathrm{pR}$ after neoadjuvant treatment with bevacizumab or cetuximab (34). Our results therefore support the role of $\mathrm{pR}$ as a surrogate marker for survival outcome after preoperative bevacizumab treatment followed by CLM resection although robust evidence of their prognostic value awaits prospective validation.

We found that in contrast to RECIST response, CT-based morphological changes correlated with $\mathrm{pR}$ after CLM resection. Consistent with previous data (19), suboptimal morphological response was significantly associated with poor $\mathrm{pR}$. However, optimal morphological response was not superior to RECIST in predicting major $\mathrm{pR}$. Morphological criteria seem to be a better surrogate marker of $\mathrm{pR}$ than RECIST response (35). However, morphological changes in the tumor do not always seem to be correlated with complete $\mathrm{pR}$ (9). Further prospective research is warranted to adequately assess the ability of morphological response to predict $\mathrm{pR}$.

This analysis also suggests that neoadjuvant or conversion treatment with chemotherapy plus bevacizumab is well tolerated. Toxicity related to this treatment strategy was generally mild and manageable, and no treatment-related deaths were reported. Safety findings are in line with phase III trials with bevacizumab plus oxaliplatin- or irinotecanbased doublet regimens $(7,8)$, with no new or unexpected signs detected. Consistent with previous randomized trials $(7,8,32)$, the most common severe adverse events were neutropenia and diarrhea.

Bleeding and wound-healing complications are common potential bevacizumab-related adverse events that can influence continuation of perioperative therapy. Consistent with previous data, we found that the addition of bevacizumab to standard chemotherapy resulted in minimal post-surgery complications (36). Indeed, no wound healing or bleeding post-surgery complications were reported according to previous experience (37) and there were no deaths due to complications of surgery. Of note, we found that bevacizumab plus chemotherapy treatment was 
continued after surgical resection in a high proportion of patients.

Neoadjuvant use of oxaliplatin-based chemotherapy has been associated with an increased risk of sinusoidal injury and subsequent postoperative morbidity $(9,17)$, while irinotecan is associated with hepatic steatosis (17). Considering the low rate of sinusoidal dilatation obtained, this analysis supports the protective effect against sinusoidal injury of adding bevacizumab to chemotherapy (11). The rate of sinusoidal injury was even lower than that reported in previous studies $(12,38)$, where only preoperative oxaliplatin-based regimens plus bevacizumab were evaluated.

The main limitation of this analysis arises from the obvious limitations of a retrospective chart review that uses existing medical data recorded for reasons other than research, including incomplete or unrecorded documentation. In particular, safety data must be considered with caution given that these came from a retrospective review of medical records where it is likely that information on adverse events may has been under-recorded. In addition, safety results must be interpreted within the context of the current safety management systems in the participating hospital. Another limitation is that the small sample analyzed for morphological response does not allow us to draw firm conclusions about its association with $\mathrm{pR}$.

Despite the above limitations, this analysis provides updated real-world data on clinical management and outcome of patients undergoing CLM resection after neoadjuvant or conversion treatment with bevacizumab plus chemotherapy. The strengths of this study include comprehensive analysis of efficacy endpoints using consistent methodology at a single Spanish University Hospital, and a relatively large and homogeneous sample of nonselected patients with CRC. Our data, although modest, are therefore particularly interesting in the context of the heterogeneity regarding methodology, resectability and tumor response criteria in this setting.

In conclusion, this analysis supports the use of bevacizumab plus chemotherapy, mainly based on oxaliplatin or irinotecan doublets, as an effective neoadjuvant or conversion treatment option for patients with CLM, with manageable toxicity, and sustains the potential prognostic value of $\mathrm{pR}$ in long-term outcome. Furthermore, this study demonstrated a low risk of postsurgical morbidity and mortality, including no bleeding events or wound-healing complications and no deaths after surgical resection.

\section{Acknowledgements}

The Authors would like to acknowledge Roche Farma S.A for supporting the study. Roche Farma kindly supported this study but this company had no role in the design of the study, in the accrual or analysis of the data, or in the preparation of the article.
The Authors also thank Cristina Vidal and Antonio Torres, from Dynamic Science, for their medical writing support, funded by Roche Farma S.A. Editorial support was provided by Miller Medical Communications Ltd, funded by grants from Roche Pharma, Spain.

\section{References}

1 Jemal A, Bray F, Center MM, Ferlay J, Ward E and Forman D: Global cancer statistics. CA Cancer J Clin 61: 69-90, 2011.

2 Kopetz S, Chang GJ, Overman MJ, Eng C, Sargent DJ, Larson DW, Grothey A, Vauthey JN, Nagorney DM and McWilliams RR: Improved survival in metastatic colorectal cancer is associated with adoption of hepatic resection and improved chemotherapy. J Clin Oncol 27: 3677-3683, 2009.

3 Okines A, Puerto OD, Cunningham D, Chau I, Van CE, Saltz L and Cassidy J: Surgery with curative-intent in patients treated with first-line chemotherapy plus bevacizumab for metastatic colorectal cancer First BEAT and the randomised phase-III NO16966 trial. Br J Cancer 101: 1033-1038, 2009.

4 Wicherts DA, de Haas RJ and Adam R: Bringing unresectable liver disease to resection with curative intent. Eur J Surg Oncol 33(Suppl 2): S42-S51, 2007.

5 Nordlinger B, Van CE, Gruenberger T, Glimelius B, Poston G, Rougier P, Sobrero A and Ychou M: Combination of surgery and chemotherapy and the role of targeted agents in the treatment of patients with colorectal liver metastases: recommendations from an expert panel. Ann Oncol 20: 985-992, 2009.

6 Wong R, Cunningham D, Barbachano Y, Saffery C, Valle J, Hickish T, Mudan S, Brown G, Khan A, Wotherspoon A, Strimpakos AS, Thomas J, Compton S, Chua YJ and Chau I: A multicentre study of capecitabine, oxaliplatin plus bevacizumab as perioperative treatment of patients with poor-risk colorectal liver-only metastases not selected for upfront resection. Ann Oncol 22: 2042-2048, 2011.

7 Saltz LB, Clarke S, Diaz-Rubio E, Scheithauer W, Figer A, Wong R, Koski S, Lichinitser M, Yang TS, Rivera F, Couture F, Sirzen $\mathrm{F}$ and Cassidy J: Bevacizumab in combination with oxaliplatin-based chemotherapy as first-line therapy in metastatic colorectal cancer: a randomized phase III study. J Clin Oncol 26: 2013-2019, 2008.

8 Hurwitz H, Fehrenbacher L, Novotny W, Cartwright T, Hainsworth J, Heim W, Berlin J, Baron A, Griffing S, Holmgren E, Ferrara N, Fyfe G, Rogers B, Ross R and Kabbinavar F: Bevacizumab plus irinotecan, fluorouracil, and leucovorin for metastatic colorectal cancer. N Engl J Med 350: 2335-2342, 2004.

9 Blazer DG, III, Kishi Y, Maru DM, Kopetz S, Chun YS, Overman MJ, Fogelman D, Eng C, Chang DZ, Wang H, Zorzi D, Ribero D, Ellis LM, Glover KY, Wolff RA, Curley SA, Abdalla EK and Vauthey JN: Pathologic response to preoperative chemotherapy: a new outcome end point after resection of hepatic colorectal metastases. J Clin Oncol 26: 5344-5351, 2008.

10 Kishi Y, Zorzi D, Contreras CM, Maru DM, Kopetz S, Ribero D, Motta M, Ravarino N, Risio M, Curley SA, Abdalla EK, Capussotti L and Vauthey JN: Extended preoperative chemotherapy does not improve pathologic response and increases postoperative liver insufficiency after hepatic resection for colorectal liver metastases. Ann Surg Oncol 17: 2870-2876, 2010. 
11 Klinger M, Tamandl D, Eipeldauer S, Hacker S, Herberger B Kaczirek K, Dorfmeister M, Gruenberger B and Gruenberger T: Bevacizumab improves pathological response of colorectal cancer liver metastases treated with XELOX/FOLFOX. Ann Surg Oncol 17: 2059-2065, 2010

12 Ribero D, Wang H, Donadon M, Zorzi D, Thomas MB, Eng C, Chang DZ, Curley SA, Abdalla EK, Ellis LM and Vauthey JN: Bevacizumab improves pathologic response and protects against hepatic injury in patients treated with oxaliplatin-based chemotherapy for colorectal liver metastases. Cancer 110: 27612767, 2007.

13 Gruenberger T, Bridgewater J, Chau I, Garcia AP, Rivoire M, Mudan S, Lasserre S, Hermann F, Waterkamp D and Adam R: Bevacizumab plus mFOLFOX-6 or FOLFOXIRI in patients with initially unresectable liver metastases from colorectal cancer: the OLIVIA multinational randomised phase II trial. Ann Oncol 26: 702-708, 2015

14 Zakaria S, Donohue JH, Que FG, Farnell MB, Schleck CD, Ilstrup DM and Nagorney DM: Hepatic resection for colorectal metastases: value for risk scoring systems? Ann Surg 246: 183191, 2007.

15 Adam R, Wicherts DA, de Haas RJ, Aloia T, Levi F, Paule B, Guettier C, Kunstlinger F, Delvart V, Azoulay D and Castaing D: Complete pathologic response after preoperative chemotherapy for colorectal liver metastases: Myth or reality? J Clin Oncol 26: 1635-1641, 2008.

16 Chan G, Hassanain M, Chaudhury P, Vrochides D, Neville A, Cesari M, Kavan P, Marcus V and Metrakos P: Pathological response grade of colorectal liver metastases treated with neoadjuvant chemotherapy. HPB 12: 277-284, 2010.

17 Rubbia-Brandt L, Giostra E, Brezault C, Roth AD, Andres A, Audard V, Sartoretti P, Dousset B, Majno PE, Soubrane O, Chaussade S, Mentha G and Terris B: Importance of histological tumor response assessment in predicting the outcome in patients with colorectal liver metastases treated with neo-adjuvant chemotherapy followed by liver surgery. Ann Oncol 18: 299304, 2007.

18 Eisenhauer EA, Therasse P, Bogaerts J, Schwartz LH, Sargent D, Ford R, Dancey J, Arbuck S, Gwyther S, Mooney M, Rubinstein L, Shankar L, Dodd L, Kaplan R, Lacombe D and Verweij J: New response evaluation criteria in solid tumours: revised RECIST guideline (version 1.1). Eur J Cancer 45: 228247, 2009.

19 Chun YS, Vauthey JN, Boonsirikamchai P, Maru DM, Kopetz S, Palavecino M, Curley SA, Abdalla EK, Kaur H, Charnsangavej $\mathrm{C}$ and Loyer EM: Association of computed tomography morphologic criteria with pathologic response and survival in patients treated with bevacizumab for colorectal liver metastases. JAMA 302: 2338-2344, 2009.

20 Chaudhury P, Hassanain M, Bouganim N, Salman A, Kavan P and Metrakos P: Perioperative chemotherapy with bevacizumab and liver resection for colorectal cancer liver metastasis. HPB 12: 37-42, 2010.

21 Vera R, Gomez DM, Lopez-Ben S, Viudez A, Queralt B, Hernandez I, Ortiz-Duran MR, Zazpe C, Soriano J, Amat I, Herrera CJ, Diaz E, Codina-Barreras A, Hernandez-Yague X, Quera A and Figueras J: Retrospective analysis of pathological response in colorectal cancer liver metastases following treatment with bevacizumab. Clin Transl Oncol 16: 739-745, 2014.
22 Terrebonne E, Smith D, Becouarn Y, Michel P, Guimbaud R, Auby D, Sa Cunha A, Ravaud A, Rouyer M, Fourrier-Réglat A, ETNA study group: Resection of colorectal cancer (CRC) metastases after bevacizumab (BV) treatment for first-line therapy: Results of the ETNA cohort study. J Clin Oncol 28(15 suppl): abstract 3594, 2010.

23 Folprecht G, Gruenberger T, Bechstein W, Raab HR, Weitz J, Lordick F, Hartmann JT, Stoehlmacher-Williams J, Lang H, Trarbach T, Liersch T, Ockert D, Jaeger D, Steger U, Suedhoff T, Rentsch A and Kohne CH: Survival of patients with initially unresectable colorectal liver metastases treated with FOLFOX/ cetuximab or FOLFIRI/cetuximab in a multidisciplinary concept (CELIM study). Ann Oncol 25: 1018-1025, 2014.

24 Abad A, Massuti B, Grávalos C, Escudero P, Guillén-Ponce C, Manzano JL, Gomez MA, Safont MJ, Gallego Plazas J, Sastre J, Pericay C, Dueñas R, López-López C, Losa F, Valladares Ayerbes M, González E, Yuste A, Carrato A, Aranda E, on behalf of the Spanish Cooperative Group for the Treatment of Digestive Tumors (TTD): Phase II trial of panitumumab plus FOLFOX4 or FOLFIRI in subjects with KRAS wild-type colorectal cancer and liver-limited disease: the PLANET study. J Clin Oncol 32(Suppl): abstract 3560, 2014.

25 Fong Y, Fortner J, Sun RL, Brennan MF and Blumgart LH: Clinical score for predicting recurrence after hepatic resection for metastatic colorectal cancer: analysis of 1001 consecutive cases. Ann Surg 230: 309-318, 1999.

26 Aoki T, Umekita N, Tanaka S, Noda K, Warabi M and Kitamura M: Prognostic value of concomitant resection of extrahepatic disease in patients with liver metastases of colorectal origin. Surgery 143: 706-714, 2008.

27 Elias D, Ouellet JF, Bellon N, Pignon JP, Pocard M and Lasser $\mathrm{P}$ : Extrahepatic disease does not contraindicate hepatectomy for colorectal liver metastases. Br J Surg 90: 567-574, 2003.

28 Leung U, Gonen M, Allen PJ, Kingham TP, DeMatteo RP, Jarnagin WR and D'Angelica MI: Colorectal cancer liver metastases and concurrent extrahepatic disease treated with resection. Ann Surg 265: 158-165, 2017.

29 Pulitano C, Bodingbauer M, Aldrighetti L, de Jong MC, Castillo F, Schulick RD, Parks RW, Choti MA, Wigmore SJ, Gruenberger $\mathrm{T}$ and Pawlik TM: Liver resection for colorectal metastases in presence of extrahepatic disease: results from an international multi-institutional analysis. Ann Surg Oncol 18: 1380-1388, 2011.

30 Elias D, Sideris L, Pocard M, Ouellet JF, Boige V, Lasser P, Pignon JP and Ducreux M: Results of R0 resection for colorectal liver metastases associated with extrahepatic disease. Ann Surg Oncol 11: 274-280, 2004.

31 Barlow AD, Nakas A, Pattenden C, Martin-Ucar AE, Dennison AR, Berry DP, Lloyd DM, Robertson GS and Waller DA: Surgical treatment of combined hepatic and pulmonary colorectal cancer metastases. Eur J Surg Oncol 35: 307-312, 2009.

32 Masi G, Loupakis F, Salvatore L, Fornaro L, Cremolini C, Cupini S, Ciarlo A, Del MF, Cortesi E, Amoroso D, Granetto C, Fontanini G, Sensi E, Lupi C, Andreuccetti M and Falcone A: Bevacizumab with FOLFOXIRI (irinotecan, oxaliplatin, fluorouracil, and folinate) as first-line treatment for metastatic colorectal cancer: a phase 2 trial. Lancet Oncol 11: 845-852, 2010.

33 Stremitzer S, Stift J, Gruenberger B, Tamandl D, Aschacher T, Wolf $\mathrm{B}$, Wrba $\mathrm{F}$ and Gruenberger $\mathrm{T}$ : KRAS status and outcome of liver resection after neoadjuvant chemotherapy including bevacizumab. Br J Surg 99: 1575-1582, 2012. 
34 Pietrantonio F, Mazzaferro V, Miceli R, Cotsoglou C, Melotti F, Fanetti G, Perrone F, Biondani P, Muscara C, Di BM, Coppa J, Maggi C, Milione M, Tamborini E and de Braud F: Pathological response after neoadjuvant bevacizumab- or cetuximab-based chemotherapy in resected colorectal cancer liver metastases. Med Oncol 32: 182, 2015.

35 Vera R, Gomez Dorronsoro ML, Ayuso JR, Figueras J, Garcia Alfonso P, Martínez-Marín V, La Casta Munoa A, Ruiz A, Safont MJ, Aparicio J, Campos JM, Camara JC, Martin-Richard M, Montagut Viladot C, Pericay C, Vieitez de Prado JM, Falco E, Jorge Fernández M, Marin $\mathrm{M}$ and Salgado Fernández $\mathrm{M}$ : Correlation between RECIST-conventional imaging techniques, morphologic response by $\mathrm{CT}$ and histopathologic response, in hepatic metastasis secondary to colorectal cancer: The AVAMET study. J Clin Oncol 33(Suppl): abstract e14656, 2015.

36 Dede K, Mersich T, Besznyak I, Zarand A, Salamon F, Baranyai ZS, Landherr L, Jakab F and Bursics A: Bevacizumab treatment before resection of colorectal liver metastases: safety, recovery of liver function, pathologic assessment. Pathol Oncol Res 19: 501-508, 2013.
37 Gruenberger B, Tamandl D, Schueller J, Scheithauer W, Zielinski C, Herbst $\mathrm{F}$ and Gruenberger T: Bevacizumab, capecitabine, and oxaliplatin as neoadjuvant therapy for patients with potentially curable metastatic colorectal cancer. J Clin Oncol 26: 1830-1835, 2008.

38 Kishi Y, Zorzi D, Contreras CM, Maru DM, Kopetz S, Ribero D, Motta M, Ravarino N, Risio M, Curley SA, Abdalla EK, Capussotti L and Vauthey JN: Extended preoperative chemotherapy does not improve pathologic response and increases postoperative liver insufficiency after hepatic resection for colorectal liver metastases. Ann Surg Oncol 17: 2870-2876, 2010.

Received January 14, 2018

Revised April 11, 2018

Accepted April 12, 2018 